\title{
Irrigação como Estratégia de Adaptação de Pequenos Agricultores às Mudanças Climáticas: aspectos econômicos ${ }^{1}$
}

\author{
Dênis Antônio da Cunha ${ }^{2}$, Alexandre Bragança Coelho ${ }^{3}$, \\ José Gustavo Féres ${ }^{4}$, Marcelo José Braga ${ }^{5}$ e Elvanio Costa de Souza ${ }^{6}$
}

Resumo: O principal objetivo deste artigo foi analisar se, dentre os pequenos agricultores brasileiros, aqueles que utilizam irrigação são menos vulneráveis que os produtores de sequeiro num contexto de mudanças climáticas. Foi desenvolvido um modelo de efeito de tratamento, por meio da técnica de Pareamento por Escore de Propensão, que permitiu identificar a adoção de irrigação e o retorno dos dois diferentes tipos de exploração agrícola simultaneamente. Foram utilizadas projeções de temperatura e precipitação para o período de 2010 a 2099, sob diferentes cenários climáticos, conforme o $4^{\mathrm{O}}$ relatório do Painel Intergovernamental de Mudanças Climáticas. Os resultados confirmaram a eficácia da irrigação como medida adaptativa. Para todos os períodos de simulação, espera-se que o valor médio da terra de pequenos produtores irrigantes seja aproximadamente o dobro do valor de sequeiro. Podese concluir que há necessidade da formulação de políticas públicas que busquem estratégias de combate aos efeitos do aquecimento global no setor, principalmente em vista da vulnerabilidade da pequena produção agrícola de sequeiro. Ademais, dada a comprovação da importância da irrigação como medida adaptativa, deve-se incentivar a expansão das políticas de crédito para a implementação dessa prática, principalmente para produtores menos capitalizados.

Palavras-chaves: Mudança climática, pequenos produtores, adaptação, irrigação.

1 Agradecemos ao Laceep (Latin American and Caribbean Environmental Program) e à Fapemig (Fundação de Amparo à Pesquisa do Estado de Minas Gerais) pela assistência financeira, ao Nemesis (Núcleo de Estudos e Modelos Espaciais Sistêmicos) e a Estáquio José Reis pela disponibilização das bases de dados climáticos e agronômicos. Agradecemos ainda aos pareceristas anônimos pela leitura atenciosa e as sugestões enviadas.

2 Doutor em Economia Aplicada. Professor Adjunto do Departamento de Economia Rural da Universidade Federal de Viçosa. E-mail: denis.cunha@ufv.br

3 Doutor em Economia Aplicada. Professor Adjunto do Departamento de Economia Rural da Universidade Federal de Viçosa. E-mail: acoelho@ufv.br

4 Doutor em Economia. Técnico de Planejamento e Pesquisa do Instituto de Pesquisa Econômica Aplicada. E-mail: jose.feres@ipea.gov.br

5 Doutor em Economia Aplicada. Professor Associado do Departamento de Economia Rural da Universidade Federal de Viçosa. E-mail: mjbraga@ufv.br

6 Doutor em Economia Aplicada. Professor Adjunto do Departamento de Economia da Universidade Federal de Viçosa. E-mail: elvanio.souza@ufv.br 
Abstract: This paper aims to analyze whether Brazilian smallholder farmers who adopt irrigation methods would be more resilient to climate change when compared to smallholders producing rainfed crops. We developed a treatment effects model, based in the Propensity Score Matching technique, which can explain irrigation adoption and net revenues simultaneously. Temperature and precipitation projections for 2010-2099 were used considering different climate scenarios according to the $4^{\text {th }}$ Assessment Report of Intergovernmental Panel on Climate Change. Results confirmed the efficiency of irrigation as an adaptive strategy. For all simulation scenarios, land values of irrigators are, on average, approximately twice the value of rainfed smallholders. It can be concluded that there is need of public policies focused on developing strategies to cope with global warming effects in the agricultural sector. Moreover, given the importance of irrigation adoption as an adaptive measure, it is needed to encourage the expansion of credit lines for irrigation investments, especially for less capitalized farmers.

Key-words: Climate change, smallholder, adaptation, irrigation.

Classificação JEL: Q12, Q54.

\section{Introdução}

As mudanças climáticas têm sido vistas como um dos maiores desafios ambientais do século XXI. A possibilidade de danos irreversíveis aos ecossistemas terrestres e de água, além de reduções no potencial de produção agrícola, desafiam pesquisadores das mais diversas áreas do conhecimento. Os efeitos das mudanças climáticas possivelmente apresentarão grande variabilidade entre as diferentes regióes do planeta e setores econômicos. Particularmente, haverá um impacto desproporcional sobre os pobres em áreas rurais, onde os meios de subsistência da maioria dependem diretamente dos recursos naturais (FISCHER et al., 2002). Há consenso entre cientistas que pequenos agricultores (e a agricultura de subsistência em geral) enfrentarão os maiores impactos negativos. De acordo com Altieri e Koohafkan (2008), esses produtores são particularmente susceptíveis devido à sua localização geográfica, baixos níveis de renda, grande dependência da agricultura (de sequeiro, principalmente) e limitada capacidade adaptativa.

Segundo Morton (2007), um quadro conceitual é necessário para compreender melhor os impactos das mudanças climáticas sobre os pequenos produtores. Tal quadro deve, necessariamente, considerar medidas de adaptação. Conforme Lobell et al. (2011), a adaptação é um fator-chave que irá moldar a gravidade futura dos impactos das mudanças climáticas na produção de alimentos. Pequenos produtores são frequentemente caracterizados por estratégias adaptativas para diminuir sua vulnerabilidade a choques climáticos, além de ações para lidar com os impactos ex-post. Com relação à America Latina, Magrin et al. (2007) e Seo (2011) afirmam que a irrigação é uma das mais importantes medidas adaptativas em resposta às mudanças climáticas.

De acordo com Dillon (2011), projetos de irrigação de pequena escala podem gerar diversos benefícios, particularmente em termos de eficiência, baixos custos de participação e mais influência sobre a gestão dos recursos hídricos. Ademais, a irrigação pode ser uma poderosa medida adaptativa no Brasil devido à disponibilidade de água e solos adequados. O País detém 12\% das reservas de água doce do planeta (MINISTÉRIO DO MEIO AMBIENTE - MMA, 2006) e possui aproximadamente 30 milhões de hectares que se configuram como solos potencialmente aptos para o desenvolvimento sustentável da agricultura irrigada (dos quais apenas 4,4 milhões de hec- 
tares estão em produção com técnicas e sistemas de irrigação) (INSTITUTO BRASILEIRO DE GEOGRAFIA E ESTATÍSTICA - IBGE, 2006).

Nesse contexto, o presente artigo examina se os pequenos agricultores que utilizam irrigação são menos vulneráveis que os produtores de sequeiro num contexto de mudanças climáticas. Este estudo desenvolve um modelo de efeito de tratamento que permite identificar a adoção de irrigação e os retornos dos dois diferentes tipos de produtores simultaneamente. Dessa forma, analisa-se como a variabilidade climática influencia a adoção de irrigação e impacta o rendimento de irrigantes e de produtores de sequeiro, considerando propriedades agrícolas com, no máximo, 10 hectares. Deve-se enfatizar que a adoção da irrigação e o retorno dos dois tipos de produtores (irrigantes ou não) vão ser considerados sob a ótica agronômica, socioeconômica e climática. Ademais, a variável a ser utilizada no retorno dos produtores é o valor da terra.

A análise é oportuna, pois a discussão a respeito das especificidades de pequenos produtores e sistemas de subsistência ainda tem sido muito pouco explorada na literatura (MORTON, 2007). No Brasil, o estudo de agricultores de pequena escala é particularmente importante, pois aproximadamente $48 \%$ dos estabelecimentos agrícolas do País têm, no máximo, 10 hectares; se for considerada a agricultura de subsistência, esse valor pode chegar a $84 \%$. Além disso, a agricultura de subsistência representa $75 \%$ da força de trabalho agrícola e é responsável por aproximadamente $10 \%$ do PIB nacional (GUILHOTO, 2007; LINDOSO et al., 2011).

Em adição, a importância de estudar ações de adaptação tem crescido rapidamente desde os anos 2000, já que é cada vez mais improvável que as mudanças climáticas poderão ser evitadas somente com esforços de mitigação (INTERNATIONAL PANEL ON CLIMATE CHANGE - IPCC, 2007; MARGULIS e DUBEUX, 2010; SEO, 2011). Ao considerar a irrigação como medida adaptativa, torna-se possível obter um entendimento mais preciso dos impactos das mudanças climáticas no setor agrícola nacional.
Sendo assim, pode-se contribuir mais efetivamente para futuras políticas públicas que visem o desenvolvimento de estratégias para combater os efeitos adversos, principalmente sobre os sistemas agrícolas de subsistência.

Os principais estudos que analisaram a agricultura brasileira (SIQUEIRA et al., 1994; SANGHI et al., 1997; NOBRE et al., 2005; ÁVILA et al., 2006; FÉRES et al.; 2008; PINTO e ASSAD, 2008) são unânimes ao afirmar que as mudanças climáticas causarão impacto líquido negativo para o País em médio e longo prazos. Há concordância também que as diversas regiões serão afetadas distintamente, o que está diretamente relacionado à substancial variação das condições edafoclimáticas ao longo do território nacional. No entanto, ainda são poucos os trabalhos que analisaram os impactos das mudanças climáticas globais sobre o setor agrícola nacional considerando explicitamente a potencialidade das medidas de adaptação. Portanto, os estudos deixaram de seguir a tendência expressa na literatura internacional sobre o tema, que é de reconhecimento às estratégias adaptativas como fonte de redução dos impactos.

Feitas essas considerações, o artigo desenvolve-se em quatro seções. Na próxima são descritos os aspectos metodológicos. $\mathrm{Na}$ terceira seção há uma detalhada descrição das variáveis e da fonte dos dados utilizados. Na seguinte, os resultados empíricos são apresentados, destacando-se as simulações para os cenários futuros de mudança climática. As conclusões são fornecidas na seção final.

\section{Metodologia}

Este estudo testa a hipótese de que a adoção de irrigação por pequenos agricultores tende a reduzir sua vulnerabilidade às mudanças climáticas. Assume-se que a decisão do produtor é tomada num processo de maximização de benefícios que garante que somente sejam observadas escolhas ótimas, independentemente de que tipo de técnica tenha sido efetivamente praticada 
(irrigação ou sequeiro). Assim, a decisão de irrigar é uma ação de otimização, influenciada pelo ambiente no qual o produtor se encontra, isto é, suas características pessoais, condição econômica, fatores climáticos etc. Em outras palavras, a escolha entre praticar agricultura de sequeiro ou irrigada não pode ser analisada como uma decisão aleatória, visto que o produtor somente será um irrigante se essa forma de produção for mais lucrativa que a primeira. Caracteriza-se, assim, a existência de autosseleção, e a simples comparação entre os resultados das duas formas de exploração agrícola resultaria numa superestimação da efetividade da técnica.

Para lidar com o problema da autosseleção, foi utilizado, neste estudo, o modelo de Efeito de Tratamento, cujas estimativas foram obtidas a partir da técnica conhecida como Pareamento por Escore de Propensão - PSM (Propensity Score Matching). Essa metodologia foi inicialmente desenvolvida por Rosenbaum e Rubin (1983). Neste artigo, foi adotado o procedimento padrão descrito em Heckman e Robb (1986), Heckman et al. (1997; 1998) e Bento et al. (2007).

Seguindo Bento et al. (2007), seja $Y_{1}$ o resultado potencial para "indivíduos tratados", que é o valor da terra num determinado município no qual há área irrigada, e $Y_{0}$ o resultado potencial que teria sido obtido no mesmo município caso a irrigação não houvesse sido praticada. Fala-se em "resultado potencial", pois apenas um deles $\left(Y_{1}\right.$ ou $\left.Y_{0}\right)$ é observado para cada município. Considera-se, ainda, que $D=1$ representa os municípios com áreas irrigadas e $D=0$ aqueles cuja produção agrícola é exclusivamente de sequeiro. Por fim, $X$ é um vetor de características observáveis que afetam tanto a adoção de irrigação quanto o valor da terra. $\mathrm{O}$ valor da terra foi utilizado para representar a lucratividade, pois, segundo Mendelsohn et al. (1994), representa o valor presente do fluxo das rendas líquidas futuras. Conforme esses autores, valores da terra proporcionam melhor medida de análise dos efeitos do clima, pois refletem a expectativa de receita líquida em muitos anos, considerando que a terra é sempre utilizada para a atividade mais lucrativa.
O efeito do uso de irrigação sobre a lucratividade agrícola, medida pelo valor da terra, é o resultado de interesse. Ele é calculado pelo efeito médio de estar em um município com áreas irrigadas em relação a outro observacionalmente equivalente, que pratica produção de sequeiro, considerando as características presentes no vetor $X$. Formalmente, o parâmetro de interesse é:

$$
\Delta T T=E\left(Y_{1}-Y_{0} \mid D=1\right)
$$

em que $\Delta T T$ refere-se ao "efeito médio do tratamento sobre os tratados", ou seja, o impacto da irrigação sobre o valor da terra daqueles que efetivamente irrigaram.

Segundo Bento et al. (2007), o método de pareamento por escore de propensão consiste em obter uma proxy para $Y_{0}$, uma vez que $Y_{0}$ não é observado para os municípios irrigantes, ou seja, para $D=1$ (e vice-versa). Essa proxy é chamada de contrafactual, ou seja, o que teria resultado no caso de produção de sequeiro. $\mathrm{O}$ estimador de escore de propensão será definido como um estimador para $E\left(Y_{0} \mid D=1\right)$, usando um subconjunto adequado dos dados de $D=0$. O método consiste em parear cada observação tratada com um ou mais não tratados, que sejam observacionalmente semelhantes, utilizando as variáveis presentes em $X$ para identificar a similaridade. Esta metodologia é válida se os resultados não são dependentes do processo de seleção, condicional aos valores de $X$. Como demonstrado por Rosenbaum e Rubin (1983), essa condição de independência é válida, condicional ao escore de propensão, $P[X]$. Dessa forma, permite-se a obtenção de um contrafactual para o grupo de tratados, de modo que qualquer diferença entre irrigantes e produtores de sequeiro será atribuída ao efeito da irrigação.

A estimação é realizada em três estágios. No primeiro, um modelo Probit da adoção de irrigação é estimado. Os coeficientes estimados são usados para calcular o escore de propensão, $P[X]$, que é a probabilidade de irrigar para cada observação. No segundo estágio, utilizando os valores do escore de propensão, os dados são 
organizados em seus respectivos grupos, ou seja, os municípios com uso de irrigação (tratados) e aqueles com produção de sequeiro, mas que têm características similares aos irrigantes (grupo de controle). Na última etapa do trabalho, estima-se um contrafactual para cada observação tratada $\left(Y_{1} \mid D=1, P[X]\right)$, baseando-se em $\left(Y_{0} \mid D=0, P[X]\right)$. Para a estimação do contrafactual, foi utilizado o método kernel, conforme sugerido por Frölich (2004). Portanto, o efeito do tratamento sobre o tratado, ou seja, o impacto da irrigação sobre o valor da terra de irrigantes, é dado por:

$$
\begin{aligned}
\Delta T T & =\left\{Y_{1}-Y_{0} \mid D=1\right\} \\
& =E\left[E\left\{Y_{1}-Y_{0} \mid D=1, P[X]\right\}\right] \\
& =E\left[E\left\{Y_{1} \mid D=1, P[X]\right\}-E\left\{Y_{0} \mid D=0, P[X]\right\} \mid D=1\right]
\end{aligned}
$$

Como o objetivo do estudo é verificar se a irrigação reduz a vulnerabilidade dos pequenos produtores, o valor de $\Delta T T$ foi calculado para as variáveis observadas no período atual e também considerando os valores de médio e longo prazo das condições climáticas. Para as simulações dos impactos da mudança do clima, foram utilizadas projeções de temperatura e precipitação para três períodos de tempo: 2010 a 2039, 2040 a 2069 e 2070 a 2099. A descrição completa das variáveis utilizadas no estudo é apresentada na próxima seção.

\section{Descrição dos $\operatorname{dados}^{7}$}

Para compor o vetor $X$, foram utilizadas variáveis climáticas, agronômicas e socioeconômicas, com base nos estudos de Negri et al. (2005), He et al. (2007), Kurukulasuriya e Mendelsohn (2007), Mendelsohn e Seo (2007), Seo e Mendelsohn (2008 a, b, c), Seo $(2010,2011)$ (Tabela 1). A unidade de observação foi Áreas Mínimas Comparáveis (AMC), que se refere à área agregada do menor número de municípios necessários para garantir comparações de uma mesma área geográfica entre diferentes anos censitários, não se tratando

\footnotetext{
7 Andersen e Reis (2007) foram responsáveis por uma parte considerável da compatibilidade do banco de dados utilizado neste estudo.
}

de uma divisão política ou administrativa ${ }^{8}$. Desde que as AMC's representam observações em nível municipal, para simplificar a exposição, optou-se por continuar utilizando o termo município.

As variáveis socioeconômicas e aquelas relacionadas ao uso de irrigação e disponibilidade de recursos hídricos foram obtidas no Censo Agropecuário 2006, disponibilizado pelo IBGE. Procurou-se caracterizar os municípios em termos do acesso de produtores à informação e ao crédito, esse último representado pelo valor dos financiamentos obtidos pelos agricultores por meio do Programa Nacional de Fortalecimento da Agricultura Familiar (Pronaf). Utilizou-se ainda uma dummy representativa dos municípios do Nordeste, já que essa região é a que possui o maior número de estabelecimentos com até 10 hectares. Ressalta-se que a distinção do Nordeste é importante, pois é a região para qual se espera os impactos mais adversos das mudanças climáticas, principalmente em relação aos pequenos produtores agrícolas.

As características agronômicas dos municípios foram obtidas por meio do Núcleo de Estudos e Modelos Espaciais Sistêmicos (Nemesis). Essas variáveis foram criadas por meio da sobreposição de limites georreferenciados de cada município sobre os dados de características agronômicas municipais. Essas variáveis caracterizam os municípios em termos do potencial agrícola do solo, potencial de erosão, altitude e disponibilidade de recursos hídricos.

\footnotetext{
8 Idealmente, deveriam ser utilizados dados em nível de produtor para cada variável. No entanto, o IBGE apenas disponibiliza esses dados, sem identificação das coordenadas geográficas (latitude e longitude), para preservar a privacidade dos produtores que responderam aos questionários do Censo. Diante disso, não é possível atribuir valores das variáveis climáticas para cada produtor. A opção por essa unidade deveu-se à necessidade de compatibilizar informações disponíveis em malhas municipais de diferentes anos. Por exemplo, as informações agronômicas fornecidas pela Embrapa (tipo de solo etc.) estavam disponíveis na malha municipal IBGE de 1996, enquanto os dados do Censo estavam na malha 2006. Tal agregação já foi utilizada nos estudos de Anderson e Reis (2007) e Féres et al. (2008 e 2009).
} 
Tabela 1. Descrição das variáveis utilizadas no estudo

\begin{tabular}{|c|c|}
\hline Variáveis & Descrição \\
\hline \multicolumn{2}{|l|}{ Condições climáticas } \\
\hline Temperatura Verão & Temperatura média de verão $\left({ }^{\circ} \mathrm{C}\right)(1961-1990)$. \\
\hline Precipitação Verão & Precipitação média de verão (mm) (1961-1990). \\
\hline Temperatura Inverno & Temperatura média de inverno $\left({ }^{\circ} \mathrm{C}\right)(1961-1990)$. \\
\hline Precipitação Inverno & Precipitação média de inverno (mm) (1961-1990). \\
\hline Variabilidade Temperatura & Variância da temperatura (1961-1990). \\
\hline Variabilidade Precipitação & Variância da precipitação (1961-1990). \\
\hline \multicolumn{2}{|l|}{ Condições agronômicas } \\
\hline Recursos Hídricos & Número de estabelecimentos agropecuários do município com recursos hídricos. \\
\hline Alto Potencial Agrícola & Proporção da área de solo do município com potencialidade agrícola na classe média/alta. \\
\hline Baixo Potencial Agrícola & Proporção da área de solo do município com potencialidade agrícola na classe baixa. \\
\hline Potencial Erosão & Proporção da área do município com limitação acentuada de erosão. \\
\hline Baixa Altitude & Proporção de área do município com altitude de 0 a 99 metros. \\
\hline Elevada Altitude & Proporção de área do município com altitude de 500 a 799 metros. \\
\hline \multicolumn{2}{|c|}{ Condições socioeconômicas } \\
\hline Acesso internet & Número de estabelecimentos agropecuários no município com acesso à internet. \\
\hline Ensino Superior & $\begin{array}{l}\text { Número de estabelecimentos agropecuários no município cujo nível de instrução da pessoa que } \\
\text { dirige o estabelecimento é o superior. }\end{array}$ \\
\hline Nordeste & Variável dummy que assume o valor 1 se o município está localizado na região Nordeste. \\
\hline Pronaf & $\begin{array}{l}\text { Valor dos financiamentos obtidos pelos agricultores por meio do Programa Nacional de } \\
\text { Fortalecimento da Agricultura Familiar (1.000 R\$). }\end{array}$ \\
\hline Valor Terra & Valor médio da terra nas $\mathrm{AMC}^{\prime} \mathrm{s}(1.000 \mathrm{R} \$)$. \\
\hline
\end{tabular}

Fonte: Cptec/Inpe, CRU, IBGE, Ipeadata.

As informações sobre temperatura e precipitação para o período corrente foram extraídas da base de dados CL 2.0 10' do Climate Research Unit - CRU/University of East Anglia. Os valores se referem à media do período de 1961-1990. Dados mensais foram utilizados para criar duas médias sazonais: dezembro a fevereiro (verão) e junho a agosto (inverno). Essa especificação é indicada, pois mantém a tendência de variação intra-anual. Além disso, supondo que a relação entre as variáveis climáticas e a produtividade das culturas seja caracterizada por não linearidades, incluiu-se também os termos quadráticos dessas variáveis. A unidade original desses dados era grid'. Por meio do programa ArcGis (versão 9.3), os pontos de grid foram unidos às fronteiras

9 Um grid é caracterizado como cada célula de um modelo de dados espaciais, as quais contêm um valor de atributo e coordenadas de localização. Tal modelo define espaço municipais usando suas coordenadas de latitude e longitude, gerando médias de temperatura e precipitação para cada município ${ }^{10}$.

Utilizaram-se também medidas de variância da temperatura e da precipitação para o período base (1961-1990), o que pode ser considerado um avanço em termos dos trabalhos já realizados para o Brasil. Essas variáveis foram incluídas, pois o foco apenas em valores médios pode levar a conclusões limitadas sobre a influência do clima na agricultura (MEARNS et al., 1997). De acordo com Negri et al. (2005), a mudança climática certamente irá afetar mais que o primeiro momento

\footnotetext{
como uma matriz de células de mesmo tamanho - os grids - organizadas em linhas e colunas.

10 A construção de variáveis climáticas foi realizada pelo Núcleo de Estudos e Modelos Espaciais Sistêmicos (Nemesis).
} 
Tabela 2. Resumo dos cenários climáticos A1B e A2, média para o Brasil

\begin{tabular}{|c|c|c|c|c|}
\hline & Período atual & 2020 & 2050 & 2080 \\
\hline \multicolumn{5}{|c|}{ Temperatura Verão $\left({ }^{\circ} \mathrm{C}\right)$} \\
\hline $\mathrm{A} 1 \mathrm{~B}$ & 24,5 & $25,5(+1.0)$ & $26,4(+1.9)$ & $27,5(+3.0)$ \\
\hline A2 & 24,5 & $25,4(+0.9)$ & $26,5(+2.0)$ & $27,9(+3.4)$ \\
\hline \multicolumn{5}{|c|}{ Temperatura Inverno $\left({ }^{\circ} \mathrm{C}\right)$} \\
\hline A1B & 20,2 & $21,6(+1.4)$ & $22,6(+2.4)$ & $23,6(+3.4)$ \\
\hline $\mathrm{A} 2$ & 20,2 & $21,5(+1.3)$ & $22,5(+2.3)$ & $24,1(+3.9)$ \\
\hline \multicolumn{5}{|c|}{ Precipitação Verão $(\mathrm{mm})$} \\
\hline A1B & 167 & $164(-1.8 \%)$ & $167(0.0 \%)$ & $168(+0.6 \%)$ \\
\hline $\mathrm{A} 2$ & 167 & $164(-1.8 \%)$ & $166(-0.6 \%)$ & $168(+0.6 \%)$ \\
\hline \multicolumn{5}{|c|}{ Precipitação Inverno $(\mathrm{mm})$} \\
\hline A1B & 56 & $63(+11.1 \%)$ & $63(+11.1 \%)$ & $63(+11.1 \%)$ \\
\hline $\mathrm{A} 2$ & 56 & $64(+12.5 \%)$ & $62(+9.7 \%)$ & $63(+11.1 \%)$ \\
\hline
\end{tabular}

Fonte: CPTEC/Inpe.

das distribuições de temperatura e precipitação, o que justifica a inclusão da variância.

É importante ressaltar que a opção por considerar apenas temperatura e precipitação de verão e inverno, ao invés das quatro estações do ano, foi baseada nos estudos de Seo e Mendelsohn (2008a) e Seo (2010 e 2011). Segundo esses autores, tal especificação é mais adequada a análises referentes à América do Sul, já que nessa região as quatro estações não são tão bem definidas quanto no hemisfério Norte. Dessa forma, espera-se que verão e inverno captem melhor os efeitos das mudanças climáticas ${ }^{11}$.

Para as projeções de temperatura e precipitação, dados médios de 10 Modelos de Circulação Geral (MCG's) foram utilizados. Consideraram-se dois cenários climáticos, A1B e A2, conforme o $4^{\circ}$ Relatório de Avaliação do IPCC (2007) ${ }^{12}$. A opção

11 No entanto, diversas especificações, incluindo também as outras estações, foram testadas. Os modelos estimados, em geral, apresentaram poucos coeficientes estatisticamente significativos, confirmando sua baixa adequação ao caso brasileiro.

12 De acordo com Margulis e Dubeux (2010), os cenários do IPCC são roteiros mais ou menos detalhados sobre o possível comportamento da economia mundial nas próximas décadas, para que se possa quantificar as prováveis emissões de GEE's. Cada cenário pressupõe um grau de crescimento econômico e seus possíveis níveis de utilização de combustíveis fosseis, desmatamento etc. Dado um cenário particular, os Modelos de Circulação Geral (MCG's) “traduzem” os níveis de emissões de GEE’s pela utilização dos cenários A1B e A2 foi baseada no estudo de Le Quéré et al. (2009), que demonstra que os padrões atuais de emissão de GEE's estão mais próximos aos cenários A1/A2 do que aos cenários $\mathrm{B}$, os quais, por sua vez, são considerados não realistas no período atual. Ademais, como o $4^{\mathrm{o}}$ Relatório do IPCC (2007) demonstra, para o período de 2020-2050 há muito menos diferenças climáticas entre cenários de emissões do que entre modelos climáticos para o mesmo cenário.

Para cada cenário climático, foram gerados dados para quatro períodos de tempo: 1961-1990 (doravante chamado "período atual"), 2010-2039 (“2020”), 2040-2069 (“2050”) e 2070-2099 (“2070”). Esses dados foram disponibilizados pelo Centro de Previsão de Tempo e Estudos Climáticos/ Instituto Nacional de Pesquisas Espaciais CPTEC/Inpe. Na Tabela 2, há um sumário dos cenários climáticos considerados.

em mudanças de temperatura, precipitação, radiação solar, elevação do nível do mar etc. Os níveis de emissões de GEE's relacionados em cada cenário utilizado podem ser vistos na Tabela A1 do Anexo. Mais detalhes sobre os cenários podem ser obtidos em: http://www.ipcc-data.org/ ddc_co2.html. 
Figura 1. Áreas mínimas comparáveis (AMC's) excluídas e consideradas na pesquisa

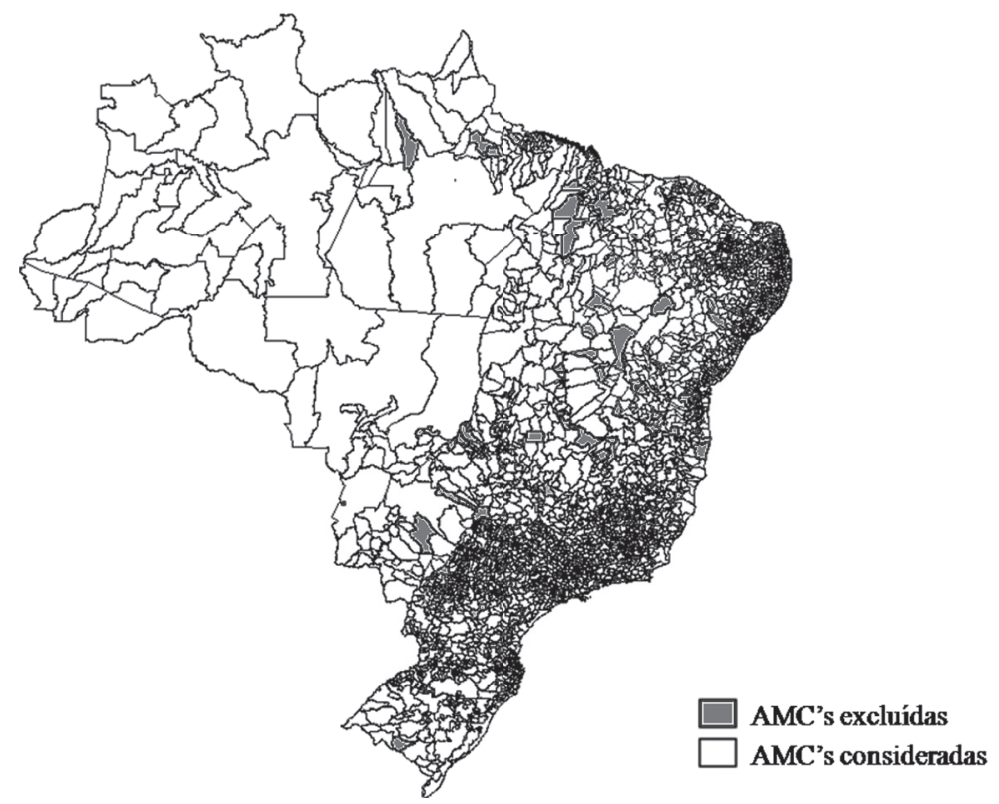

Fonte: Ipeadata, com dados trabalhados pelo autor.

A base de dados continha, originalmente, 3.659 observações. No entanto, foram desconsideradas as áreas urbanas, já que, segundo Schlenker et al. (2005), a forte influência da urbanização sobre os valores da terra nessas regióes poderia causar viés. Além disso, foram retiradas as localidades para as quais o banco de dados não registrava os valores da terra, além de outros dados considerados questionáveis (por exemplo, observações cuja área agrícola reportada era maior que a área total do município). A base de dados final foi composta por 3.123 observações. A Figura 1 apresenta a distribuição das AMC's mantidas e excluídas. Pode-se observar que, mesmo após as exclusões, a amostra representa adequadamente todas as regiões do País.

\section{Resultados e discussão}

Para dar início à análise, podem-se observar as estatísticas descritivas das variáveis, considerando separadamente os dois tipos de produção agrícola (Tabela 3).
Em termos climáticos, diferenças entre a produção irrigada e a de sequeiro foram observadas apenas para as variáveis de precipitação, já que a temperatura não apresentou médias estatisticamente distintas. Irrigantes estiveram expostos a menor volume de chuva, resultado que é mais expressivo no verão. Portanto, pode-se afirmar que, quando a chuva é abundante, produtores tendem a praticar agricultura de sequeiro; porém, à medida que a precipitação se torna escassa, há gradual troca para os sistemas irrigados. Constatações semelhantes são encontradas no estudo de Seo (2011), que analisou a irrigação na América Latina. É importante ressaltar que tanto irrigantes quanto produtores de sequeiro estiveram expostos à alta variabilidade da precipitação e baixa variação de temperatura.

Diferenças podem ser igualmente verificadas em termos das variáveis agronômicas e socioeconômicas. As áreas com produção de sequeiro, em geral, tiveram menor acesso aos recursos hídricos e estavam localizadas em municípios com baixa qualidade do solo. Por outro lado, irrigantes tiveram maior acesso a tecnologias de informação, 
Tabela 3. Estatísticas descritivas por tipo de produção

\begin{tabular}{|c|c|c|c|c|}
\hline \multirow{2}{*}{ Variáveis } & \multicolumn{2}{|c|}{ Irrigantes } & \multicolumn{2}{|c|}{ Produtores de sequeiro } \\
\hline & Média & Desvio Padrão & Média & Desvio Padrão \\
\hline \multicolumn{5}{|l|}{ Condições climáticas } \\
\hline Temperatura Verão & 24,51 & 1,97 & 24,45 & 2,02 \\
\hline Precipitação Verão & 165,28 & 73,27 & 184,21 & 75,71 \\
\hline Temperatura Inverno & 20,17 & 3,81 & 20,19 & 3,93 \\
\hline Precipitação Inverno & 53,02 & 50,46 & 59,27 & 55,13 \\
\hline Variabilidade Temperatura & 3,76 & 2,91 & 3,66 & 2,72 \\
\hline Variabilidade Precipitação & $5.295,29$ & $3.483,17$ & $6.032,62$ & $3.939,46$ \\
\hline \multicolumn{5}{|l|}{ Condições agronômicas } \\
\hline Recursos Hídricos & 427,40 & 738,10 & 122,05 & 165,03 \\
\hline Alto Potencial Agrícola & 0,12 & 0,27 & 0,08 & 0,24 \\
\hline Baixo Potencial Agrícola & 0,55 & 0,42 & 0,60 & 0,42 \\
\hline Potencial Erosão & 0,42 & 0,35 & 0,38 & 0,38 \\
\hline Baixa Altitude & 0,12 & 0,29 & 0,15 & 0,33 \\
\hline Alta Altitude & 0,30 & 0,33 & 0,26 & 0,35 \\
\hline \multicolumn{5}{|l|}{ Condições socioeconômicas } \\
\hline Acesso internet & 6,99 & 23,78 & 1,76 & 3,83 \\
\hline Ensino Superior & 6,95 & 12,61 & 1,93 & 2,91 \\
\hline Pronaf & 270,88 & 807,74 & 73,09 & 161,48 \\
\hline Nordeste & 81,56 & - & 18,44 & - \\
\hline Valor Terra & $10.569,96$ & $15.878,44$ & $5.057,93$ & $15.009,78$ \\
\hline
\end{tabular}

Notas: (1) As variáveis Valor Terra e Pronaf estão cotadas em R 1.000 (valores referentes ao Censo Agropecuário 2006); (2) O valor associado à variável Nordeste indica que, entre produtores da região, $81,56 \%$ praticam alguma técnica de irrigação e 18,44\% são produtores de sequeiro.

Fonte: Resultados da pesquisa.

como internet, e maior número de agricultores com formação superior. Pode-se afirmar, ainda, que esses últimos utilizaram volume superior de créditos do Pronaf. Finalmente, o valor médio da terra de irrigantes foi mais elevado. Esse é um primeiro indício de que a utilização de técnicas de irrigação, ao reduzir o risco associado a alterações no clima, entre outros benefícios, gera maiores rendimentos ao produtor, configurando-se como efetiva medida de adaptação.

Para identificar o efeito da irrigação sobre o valor da terra dos pequenos produtores, inicialmente foi realizado o cálculo dos escores de propensão, a partir de um modelo Probit. O modelo permitiu identificar como as distintas variáveis exógenas e, especialmente, os fatores climáticos, afetaram a escolha de irrigação. $\mathrm{O}$ resultado do modelo está apresentado na Tabela 4 . Considerando-se a estatística razão de verossimilhança (LR), o modelo foi estatisticamente significativo. Exceto pela variável referente à variabilidade da precipitação, todos os coeficientes das variáveis climáticas foram diferentes de zero. Dessa forma, pode-se afirmar, assim como nos estudos de Mendelsohn e Seo (2007), Kurukulasuriya et al. (2011) e Seo (2011), que a adoção de irrigação é sensível tanto à temperatura quanto à precipitação, o que valida sua modelagem como estratégia adaptativa.

Em relação às variáveis climáticas, considerações importantes podem ser feitas. Observou-se um padrão de U para a variável temperatura de verão e de $U$ invertido para a temperatura de inverno. $\mathrm{O}$ primeiro pode ser atribuído às características de verão. Nessa estação, aumentos de temperatura, em geral, são acompanhados por mais chuvas no Brasil. No caso da temperatura de inverno, o resultado é semelhante aos de Mendelsohn e Seo (2007) e de Seo (2011). Considerando-se que as decisões de irrigação 
Tabela 4. Estimativas do modelo Probit de determinação da probabilidade de irrigar para pequenos produtores

\begin{tabular}{lcc}
\hline \multicolumn{1}{c}{ Variáveis } & Coeficiente & Desvio Padrão \\
\hline Temperatura Verão & $-1,039^{*}$ & 0,548 \\
Temperatura Verão $^{2}$ & $0,017^{*}$ & 0,010 \\
Precipitação Verão $^{*}-0,005^{* * *}$ & 0,001 \\
Temperatura Inverno & $0,517^{* *}$ & 0,245 \\
Temperatura Inverno ${ }^{2}$ & $-0,009^{*}$ & 0,005 \\
Precipitação Inverno & $-0,005^{* * *}$ & 0,001 \\
Variabilidade Temperatura & $0,084^{*}$ & 0,050 \\
Variabilidade Precipitação & $0,000^{\mathrm{NS}}$ & 0,000 \\
Recursos Hídricos & $0,002^{* * *}$ & 0,000 \\
Alto Potencial Agrícola & $0,254^{* *}$ & 0,120 \\
Baixo Potencial Agrícola & $0,012^{\mathrm{NS}}$ & 0,081 \\
Potencial Erosão & $0,206^{* *}$ & 0,083 \\
Baixa Altitude & $0,115^{\mathrm{NS}}$ & 0,134 \\
Elevada Altitude & $0,141^{\mathrm{NS}}$ & 0,098 \\
Acesso Internet & $0,031^{* * *}$ & 0,010 \\
Ensino Superior & $0,065^{* * *}$ & 0,013 \\
Nordeste & $-0,244^{\mathrm{NS}}$ & 0,152 \\
Pronaf & $0,000^{\mathrm{NS}}$ & 0,000 \\
Intercepto & $9,054^{*}$ & 4,654 \\
\hline Not & $-0,0000 ;)$ A &
\end{tabular}

Notas: (1) A estatística LR estimada foi 240,56 com P-valor = 0,0000; (2) Desvio padrão obtido após a correção de heterocedasticidade; (3) $\left(^{* * *}\right),\left({ }^{* *}\right)$ e $\left(^{*}\right)$ indicam significância a 1\%, $5 \%$ e 10\%, respectivamente; $\left({ }^{\mathrm{NS}}\right)$ indica não significância.

Fonte: Resultados da pesquisa.

são tomadas antes da estação de crescimento das plantas, baseando-se nas condições do tempo esperadas, há maior pré-disposição a irrigar à medida que se espera temperaturas mais elevadas. Ressalta-se, ainda que quando a temperatura está alta, faz-se a opção por irrigar em períodos de temperatura mais baixa (à noite, por exemplo), de modo a garantir a eficiência da técnica.

Os sinais negativos da precipitação confirmam seu relacionamento inverso com a probabilidade de irrigar. Verificou-se que a variância da precipitação não foi significativa, diferentemente da variância da temperatura. O coeficiente dessa última variável apresentou sinal positivo, indicando que os pequenos produtores são avessos ao risco de mudanças bruscas de temperatura. Por esses resultados, pode-se afirmar que, nos estabelecimentos agropecuários com até 10 hectares, a irrigação é praticada também como forma de minimizar os riscos associados ao estresse térmico.
No que se refere às características agronômicas e socioeconômicas, foi constatada a importância da disponibilidade de recursos hídricos, de solos aptos para a prática agrícola e do acesso à informação. Com relação a esse último aspecto, o conhecimento técnico, representado pela variável relativa ao ensino superior, mostrou-se positivamente relacionado à prática de irrigação. Ademais, o coeficiente da variável representativa do acesso à internet também foi positivo. A internet é uma importante ferramenta de busca dos mais diferentes tipos de informações, entre elas, as mudanças climáticas. Produtores podem acessar previsões de temperatura e precipitação para vários períodos de tempo, facilitando sua tomada de decisões. Além disso, tem se tornado comum a assistência técnica remota, em que as informações são transmitidas diariamente aos irrigantes por meio da internet. Controla-se, à distância, o manejo da irrigação do dia atual e calcula-se a probabilidade de irrigação para dias futuros. Para 
Tabela 5. Estimativas do efeito das mudanças climáticas sobre o valor médio da terra de irrigantes e produtores de sequeiro

\begin{tabular}{ccccc}
\hline Variável de resposta & Irrigantes & Produtores de sequeiro & Diferença & P-valor \\
\hline Período Atual & & & & \\
\hline Valor Terra & $8.694,96$ & $12.153,73$ & $-3.459,60^{* * *}$ & 0,0000 \\
\hline Cenário A1B & & & & 0,0000 \\
\hline Valor Terra (2020) & $10.578,71$ & $4.953,43$ & 0,0000 \\
Valor Terra (2050) & $10.575,46$ & $4.891,44$ & $5.684,01^{* * *}$ & 0,0000 \\
Valor Terra (2080) & $10.567,44$ & $4.837,87$ & $5.729,57^{* * *}$ & 0,0000 \\
\hline Cenário A2 & & & & 0,0000 \\
Valor Terra (2020) & $10.578,71$ & $4.973,97$ & $5.604,72^{* * *}$ & 0,0000 \\
Valor Terra (2050) & $10.578,00$ & $4.989,72$ & $5.715,28^{* * *}$ & \\
Valor Terra (2080) & $10.573,73$ & $4.858,12$ & & \\
\hline
\end{tabular}

(1) A variável Valor Terra está cotada em 1.000 R\$ (valores referentes ao Censo Agropecuário 2006); (2) P-valor baseado no erro padrão calculado por bootstrap; (3) (***) indica significância a $1 \%$.

Fonte: Resultados da pesquisa.

dar a orientação, a assistência técnica igualmente recebe informações via internet.

A variável indicadora de produtores localizados no Nordeste não foi estatisticamente significativa. Embora não esperado, acredita-se que esse resultado pode estar relacionado à baixa eficácia das políticas de irrigação direcionadas à região. Coelho Neto (2009) ressalta que, apesar de sempre ter havido prioridade da Política Nacional de Irrigação em relação ao Nordeste, os resultados efetivos, em termos de ampliação do espaço irrigado, assinalam pequeno avanço quando comparado com as demais regiões do País. O autor explica ainda que a expansão da área irrigada no Nordeste é insatisfatória em relação às potencialidades concretas identificadas pelos diversos estudos produzidos pelo governo.

Esperava-se que a variável Pronaf tivesse efeito positivo sobre a decisão de irrigar, já que se trata de importante fonte de crédito para a aquisição de máquinas, equipamentos ou infraestrutura de produção. No entanto, o coeficiente estimado não foi estatisticamente significativo, o que pode indicar baixa expressividade de créditos do Pronaf destinados especificamente a projetos de irrigação.

Uma vez discutidos os fatores associados à adoção de irrigação, pode-se analisar seu efeito sobre o valor da terra dos irrigantes, considerando cenários climáticos atuais e futuros.
Escores de propensão foram construídos utilizando as estimativas do modelo Probit, de modo a garantir a correspondência das características observáveis dos municípios nos grupos de tratamento e de controle. É importante enfatizar que as simulações para os cenários futuros foram realizadas alterando apenas as condições climáticas. De acordo com Seo (2011), o objetivo desse tipo de simulação é isolar os efeitos do clima de outras mudanças nas condições econômicas.

A Tabela 5 contém os valores estimados referentes ao efeito do tratamento sobre os tratados utilizando o estimador kernel $^{13}$. Pode-se verificar o valor da terra para cada período e cenário climático. As diferenças estimadas entre as duas classes de produtores são estatisticamente significativas a $1 \%$. Deve-se ressaltar que testar a significância estatística dos efeitos do tratamento e calcular o seu erro padrão não é um procedimento simples. De acordo com Caliendo e Kopeinig (2005), o problema é que a variância estimada do efeito do tratamento deve incluir a variância devida à estimativa do escore de propensão e também a ordem em que os indivíduos tratados são com-

\footnotetext{
13 Para explorar a sensibilidade das estimativas a variáveis não observadas, bem como para confirmar a robustez do modelo, foi realizado o teste dos limites de Rosenbaum. Os resultados (Tabela A3 do Anexo) indicaram alta significância do coeficiente de efeito de tratamento estimado, o que assegura a não existência de viés significativo resultante de variáveis omitidas no modelo.
} 
parados com os não tratados. Uma forma de lidar com esse problema é usar a técnica de bootstrap, como sugerido por Lechner (2002). O bootstrap busca gerar uma distribuição de frequências após a replicação de inúmeras amostras selecionadas aleatoriamente (com reposição) da amostra original e com o mesmo tamanho. Em cada etapa do procedimento há a reestimação dos resultados de modo a obter o efeito do tratamento " $\mathrm{n}$ " vezes. $\mathrm{A}$ distribuição dos valores médios aproxima a distribuição da amostra e, assim, é possível calcular um erro padrão médio que garante a precisão para as estimativas do efeito de tratamento.

Os resultados da Tabela 5 mostram que, no período atual, os retornos associados ao uso de irrigação são menores que nos sistemas de sequeiro. Acredita-se que o principal fator explicativo desse resultado, que a princípio não era esperado, seja o elevado custo da irrigação. Apesar de a irrigação aumentar a produtividade, o resultado parece indicar que, no período presente, em média, seu custo não compensa o aumento de receita. Essa conclusão é respaldada pela análise do Ministério da Integração (2008). Segundo o referido estudo, a produção irrigada de grãos, nas condições vigentes no País, tem grande dificuldade de competir com a de sequeiro, em razão dos investimentos que devem ser realizados e dos custos de administração, operação e manutenção. Esse aspecto é ainda mais complicado para pequenos produtores devido à sua reduzida capacidade de investimento.

O resultado obtido para o período atual também pode estar relacionado à natureza da irrigação praticada por parcela substancial de pequenos agricultores. De acordo com dados do Censo Agropecuário (IBGE, 2006), a técnica de irrigação mais utilizada, em $38 \%$ dos estabelecimentos com até 10 hectares, refere-se a regas manuais com a utilização de regadores, mangueiras, baldes e latões. O emprego desse tipo rudimentar de irrigação dificilmente se reflete no valor da terra.

No entanto, quando são considerados os cenários de mudanças climáticas, foi possível observar a eficácia da irrigação como medida adaptativa. Conforme discutido por Schlenker et al. (2005), os benefícios e custos (incluindo os gastos associados à implantação do sistema de irrigação) são capitalizados nos valores da terra. Dessa forma, pode-se afirmar que, frente a cenários de mudanças climáticas, os retornos obtidos com a adoção de irrigação são superiores aos custos. Esse resultado está em conformidade com os apresentados no estudo de Margulis e Dubeux (2010). Segundo os autores, a relação entre o custo de implantação de um sistema de irrigação e o benefício (medido pelas perdas evitadas) mostra-se vantajosa, variando de $2 \%$ em 2020 a $19 \%$ em 2070 na produção de milho, e de $11 \%$ a $41 \%$ na produção de arroz.

Verifica-se, ainda na Tabela 5, que a agricultura de sequeiro pode enfrentar considerável prejuízo em relação à produção irrigada. Para todos os períodos de simulação, espera-se que o valor médio da terra de pequenos produtores irrigantes seja aproximadamente o dobro do valor de sequeiro. Em relação ao valor estimado para o período atual, as perdas para a produção não irrigada poderiam chegar a cerca de $\mathrm{R} \$$ 7,2 milhões já nos próximos 30 anos (período de 2010 a 2039).

Se os pequenos produtores utilizarem irrigação, espera-se aumento do valor da terra, que pode chegar a $21,6 \%$ em 2020 e 2050 , e a $20,5 \%$ em 2080. Para a pequena produção não irrigada, são esperadas reduções do valor da terra da ordem de $59,2 \%$ em 2020 , de $59,7 \%$ em 2050 e de $60 \%$ em 2080. Seo e Mendelsohn (2008a), utilizando a modelagem hedônica tradicional, estimaram que os pequenos produtores da América do Sul poderiam ter perdas de até $44 \%$. Acredita-se, no entanto, que esse processo de mudança para sistemas adaptados será gradual e levará alguns anos até se completar, dependendo, inclusive de políticas públicas específicas.

Ressalta-se ainda que, se for computado o desvio padrão entre os valores da terra simulados no cenário A1B, por exemplo, no período de 2020 a 2080, verifica-se que para irrigantes o desvio é 5,8 , ao passo que, para produtores de sequeiro, é 57,8 . Esse padrão se repete para o cenário A2 e também quando são considerados diferentes 
períodos (2020-2050 e 2050-2080) ${ }^{14}$. Confirma-se, dessa forma, que os valores da terra de irrigantes têm menor variabilidade, pois, certamente, a adaptação deixa os agricultores menos sensíveis às mudanças climáticas. No caso dos produtores de sequeiro, a variabilidade é superior, indicando sua exposição maior às mudanças do clima e, consequentemente, sua vulnerabilidade. Essa explicação está em conformidade com a análise de Deressa et al. (2008). Segundo esses autores, a magnitude e taxa de variação no clima determinam o grau de exposição do setor que, por sua vez, interfere em sua vulnerabilidade, ou seja, os ganhos ou perdas esperados para a produção agrícola. Mas a sensibilidade ao clima é diretamente afetada pela capacidade adaptativa. Em outras palavras, dado um nível fixo de exposição, alta (baixa) capacidade adaptativa diminui (aumenta) a vulnerabilidade do sistema.

Esses resultados permitem a construção de um cenário de impactos das mudanças climáticas bastante diferenciado entre pequenos produtores que se adaptam e os que não o fazem. Inicialmente, sobre o aumento no valor da terra de irrigantes, acredita-se que os resultados sejam consequência dos ganhos de produtividade. Como, em geral, pequenos produtores são pouco produtivos, a utilização de irrigação tenderia a aumentar muito sua produtividade. Além disso, se esses agricultores estiverem localizados mais próximos ao meio urbano ou se realizam cultivos de maior valor de mercado, como, por exemplo, a agricultura orgânica, faz sentido que os ganhos em termos de valor da terra sejam expressivos.

Já as perdas, bastante altas, podem estar relacionadas à dependência desses produtores em relação à produção agrícola. Conforme Altieri e Koohafkan (2008), a maior parte dos modelos climáticos prevê que os prejuízos relacionados às mudanças do clima serão desproporcionalmente suportados por pequenos produtores dos países em desenvolvimento. As estimativas de Jones e Thornton (2003), por exemplo, indicam perdas na

${ }_{14}$ Os valores dos desvios padrão calculados podem ser vistos na Tabela A4 do Anexo. produção de milho de US\$ 2 bilhões por ano até 2055 na África e América Latina.

Ademais, o alto impacto pode estar associado ao fato de a maior parcela dos estabelecimentos com até 10 hectares estar localizada no Nordeste (cerca de 60\%, segundo o IBGE, 2006). Essa região será a que enfrentará os maiores efeitos negativos das alterações futuras do clima. Conforme Margulis e Dubeux (2010), as principais culturas produzidas no Nordeste, notadamente as de subsistência, devem registrar forte impacto negativo devido à elevação da temperatura e à redução da oferta de recursos hídricos; sem nenhum tipo de adaptação, as áreas de cultivo dos estados nordestinos podem ser consideravelmente reduzidas.

A confirmação de que pequenos produtores possivelmente terão impactos negativos severos pode trazer uma série de consequências adversas ao País, uma vez que, de acordo com o Censo Agropecuário (IBGE, 2006), cerca de 2,4 milhões de estabelecimentos agropecuários do Brasil têm suas atividades realizadas em áreas com, no máximo, 10 hectares. Além disso, a pequena produção agrícola é uma das principais fontes de emprego no meio rural, principalmente nas regiões Norte, Nordeste e Sul. Dessa forma, os impactos negativos previstos neste estudo podem desencadear desequilíbrios no mercado de trabalho rural, com efeitos diretos sobre os níveis de migração e o nível geral de bem-estar e segurança alimentar da sociedade.

\section{Conclusões}

No Brasil, o estudo dos impactos das mudanças climáticas sobre o setor agrícola tem ganhado visibilidade desde meados da década de 90. As distintas análises indicam que o País terá perdas econômicas, que podem variar de valores modestos no curto prazo até grandes prejuízos no longo prazo. E esta questão é de importância estratégica, ao se considerar que a produção agropecuária responde por parcela expressiva da renda nacional e que há um grande número de pequenos agricultores cuja vulnerabilidade é alta. As 
perdas na agricultura podem trazer diversos problemas, como o aumento da pobreza rural e suas consequências nas cidades, que terão que receber milhares de migrantes. Dessa forma, este artigo procurou contribuir para a ampliação do entendimento a essas questões ao estudar os efeitos da irrigação sobre a renda de produtores com no máximo 10 hectares.

A análise dos fatores associados à adoção de irrigação no Brasil indicou que condições agronômicas, socioeconômicas e climáticas influenciam a sua prática. $\mathrm{O}$ acesso aos recursos hídricos e a existência de terras em boas condições para as atividades agrícolas são importantes. A decisão do produtor ainda é condicionada pelo seu conhecimento técnico e acesso à informação. Confirmou-se a expectativa de que a irrigação é influenciada pelas variações climáticas e, dessa forma, pode efetivamente ser modelada como estratégia adaptativa.

No que se refere ao efeito da irrigação sobre os rendimentos dos produtores, os resultados apontam duas conclusões distintas. No período atual, foram estimados retornos maiores para a produção de sequeiro. Essa resposta está diretamente ligada aos custos elevados de implantação de um sistema de irrigação, fazendo com que essa forma de exploração agrícola seja menos rentável. Ademais, isso provavelmente se dá porque muitos agricultores utilizam a prática sem se preocuparem com o manejo da água e com os demais fatores que são necessários para que se produza eficientemente com irrigação. Não obstante, quando se consideram os cenários futuros de mudanças climáticas, o resultado se inverte, ou seja, a renda dos irrigantes tende a ser crescente e mais estável. Diferente de outros estudos, que estimam perdas elevadas para toda a agricultura brasileira, este trabalho conclui que os produtores que se adaptarem podem apresentar retornos crescentes no médio e longo prazo. No entanto, uma característica da metodologia utilizada é que não é possível fazer uma avaliação mais detalhada sobre a intensidade e prazo da ocorrência dessas alterações. Esse é um ponto que deve ser considerado em estudos posteriores.
As perdas esperadas para a produção de sequeiro são consideravelmente grandes. É necessário, dessa forma, implementar programas de capacitação de pequenos agricultores, criando condições para que eles possam se adaptar. Ao mesmo tempo, esse resultado reforça a necessidade da formulação de políticas públicas que busquem estratégias de combate aos efeitos do aquecimento global no setor, já que a pequena produção agrícola de sequeiro possivelmente será bastante prejudicada. Ademais, dada a comprovação da importância da irrigação como medida adaptativa, deve-se incentivar a expansão das políticas de crédito específicas para a implementação dessa prática, principalmente para produtores menos capitalizados.

Por fim, o resultado obtido pode indicar que a irrigação brasileira necessita de maior investimento por unidade de área, uma vez que esse recurso adicional pode representar grande ganho de eficiência da prática. Esse "esforço adicional" visaria a modernização de equipamentos de irrigação e a instalação de infraestrutura para melhoria do manejo da água, sobretudo para pequenos produtores. Complementarmente, a garantia dada pela irrigação no tocante à segurança alimentar pode indicar que se justificaria a concessão de algum tipo de subsídio nos casos em que a produção objetivasse o abastecimento do mercado interno. Logicamente, esses avanços somente serão obtidos num ambiente de evolução das políticas públicas e do sistema de extensão rural.

\section{Referências bibliográficas}

ALTIERI, M. A. e KOOHAFKAN, P. Enduring Farms: Climate Change, Smallholders and Traditional Farming Communities. Penang, Malaysia: Third World Network, 2008.

ÁVILA, A. F. D., IRIAS, L. J. M. e LIMA, M. Impacto das mudanças climáticas na agricultura brasileira. Brasília: Embrapa, 2006.

BENTO, A., TOWE, C. e GEOGHEGAN, J. The effects of moratoria on residential development: evidence from a matching approach. The American Journal of Agricultural Economics, v. 89, n. 5, 1211-1218, 2007. 
CALIENDO, M. e KOPEINIG, S. Some practical guidance for the implementation of propensity score matching. Discussion paper no ${ }^{\circ}$ 1588. Bonn: IZA, 2005.

COELHO NETO, A. S. Trajetórias e direcionamentos da política de irrigação no Brasil: as especificidades da região Nordeste e do Vale do São Francisco. In: XIX Encontro Nacional de Geografia Agrária, 2009, São Paulo - SP. Anais... São Paulo: USP, 2009.

DERESSA, T., HASSAN, R. M. e RINGLER, C. Measuring Ethiopian Farmers' Vulnerability to Climate Change Across Regional States. IFPRI Discussion Paper 00806, 2008. Disponível em: <http://www.ifpri.org/publication/ measuring-ethiopian-farmers-vulnerability-climatechange-across-regional-states>. Acesso em: nov. 2012.

DILLON, A. Do differences in the scale of irrigation projects generate different impacts on poverty and production? Journal of Agricultural Economics, v. 62, n. 2, p. 474-492, 2011.

FÉRES, J., REIS, E. e SPERANZA, J. Assessing the Impact of Climate Change on the Brazilian Agricultural Sector. In: $16^{\text {th }}$ Annual EAERE Conference, 2008, Gothenburg. Proceedings of the $16^{\text {th }}$ Annual EAERE Conference. Gothenburg: EAERE, 2008.

FÉRES, J., REIS, E. e SPERANZA, J. Mudanças climáticas globais e seus impactos sobre os padrões de uso do solo no Brasil. In: XLVII Congresso da Sociedade Brasileira de Economia, Administração e Sociologia Rural, 2009, Porto Alegre - RS. Anais... Brasília: SOBER, 2009.

FISCHER, G., SHAH, M. e VAN VELTHUIZEN, $\mathrm{H}$. Climate change and agricultural vulnerability. Johannesburg: International Institute for Applied Systems Analysis to World Summit on Sustainable Development, Special Report, 2002.

FRÖLICH, M. Finite-sample properties of propensityscore matching andweighting estimators. The Review of Economics and Statistics, v. 86, n. 1, p. 77-90, 2004.

GUILHOTO, J. PIB da agricultura familiar Brasil estados. Brasília: MDA, 2007. Disponível em: <http:// portal.mda.gov.br/portal/saf/arquivos/view/ater/livros/ PIB-AFamiliar_x_Patronal-2002-2005.pdf > . Acesso em: Out. 2011.

HE, X., CAO, H. e LI, F. Econometric analysis of the determinants of adoption of rainwater harvesting and supplementary irrigation technology (RHSIT) in the semiarid Loess Plateau of China. Agricultural Water Management, v. 89, p. 243-250, 2007.

HECKMAN, J. J. e ROBB, R. Alternative method for solving the problem of selection bias in evaluating the impact of treatments on outcomes. In: Wainer, H. (Ed.). Drawing Inferences from Self-Selected Samples. SpringerVerlag, Berlin, 1986. p. 63-107.

HECKMAN, J. J., ICHIMURA, H. e TODD, P. E. Matching as an econometric evaluation estimator: evidence from evaluating a job training programme. The Review of Economic Studies, v. 64, n. 4, 605-654, 1997.

HECKMAN, J. J., ICHIMURA, H., SMITH, J. A. e TODD, P. E. Characterizing selection bias using experimental data. Econometrica, v. 66, n. 5, p. 1017-1098, 1998.

INSTITUTO BRASILEIRO DE GEOGRAFIA E ESTATÍSTICA - IBGE. Censo Agropecuário 2006: Brasil, Grandes Regiões e Unidades da Federação. Rio de Janeiro: IBGE. 2006.

INTERNATIONAL PANEL ON CLIMATE CHANGE IPCC. Climate Change 2007: Synthesis Report. Contribution of Working Groups I, II and III to the Fourth Assessment Report of the Intergovernmental Panel on Climate Change. PACHAURI, R. K. e REISINGER, A. (Eds.). Geneva, Switzerland: IPCC, 2007.

JONES, P. G. e THORNTON, P. K. The potential impacts of climate change on maize production in Africa and Latin America in 2055. Global Environmental Change, v. 13, n. 1, p. 51-59, 2003.

KURUKULASURIYA, P., KALA, N. e MENDELSOHN, R. Adaptation and climate change impacts: a structural Ricardian model of irrigation and farm income in Africa. Climate Change Economics, v. 2, n. 2, p. 149-174, 2011.

KURUKULASURIYA, P. e MENDEOSOHN, R. Endogenous irrigation: the impact of climate change on farmers in Africa. World Bank Policy Research Working Paper 4271. Washington, D. C.: World Bank, 2007.

LECHNER, M. Some practical issues in the evaluation of heterogenous labour market programmes by matching methods. Journal of the Royal Statistical Society, v. 165, p. 59-82, 2002.

LINDOSO, D. P., ROCHA, J. D., DEBORTOLI, N., PARENTE, I. C. I. P., EIRÓ, F., BURSZTYN, M. e RODRIGUES FILHO, S. Agricultura familiar e mudanças climáticas: avaliando a vulnerabilidade à seca no semiárido nordestino. In: SERÔA DA MOTTA, R., HARGRAVE, J., LUEDEMANN, G. e GUTIERREZ, M. B. S. Mudança do clima no Brasil: aspectos econômicos, sociais e regulatórios. Brasília: IPEA, 2011.

LE QUÉRÉ, C., RAUPACH, M. R., CANADELL, J. G., MARLAND, G. et al. Trends in the sources and sinks of carbon dioxide. Nature Geoscience, v. 689, p. 1-6, 2009. 
LOBELL, D. B., BURKE, M. B., TEBALDI, C., MASTRANDREA, M. D., FALCON, W. P. e NAYLOR, R. L. Prioritizing climate change adaptation needs for food security in 2030. Science, v. 319, p. 607-610, 2008.

MAGRIN, G., GARCIA, C. G., CHOQUE, D. C., GIMENEZ, J. C., MORENO, A. R., NAGY, G. J., CARLOS, N. e VILLAMIZAR, A. Latin America. In: PARRY, M. L., CANZIANI, O. F., PALUTIKOF, J. P., VAN DER LINDEN, P. J. e HANSON, C. E. (Eds.). Climate Change 2007: impacts, adaptation, and vulnerability - Contribution of Working Group II to the Fourth Assessment Report of the Intergovernmental Panel on Climate Change. Cambridge, UK: Cambridge University Press, 2007. p. 581-615.

MARGULIS, S. e DUBEUX, C. B. S. Economia da Mudança do Clima no Brasil: custos e oportunidades. São Paulo: IBEP Gráfica, 2010.

MEARNS, L., ROSENZWEIG, C e GOLDBERG, R. Mean and variance change in climate scenarios: methods, agricultural applications, and measures of uncertainty. Climatic Change, v. 35, n. 4, p. 367-396, 1997.

MENDELSOHN, R., NORDHAUS, W. e SHAW, D. The impact of global warming on agriculture: Ricardian analysis. The American Economic Review, v. 84, n. 4, p. 753-771, 1994.

MENDELSOHN, R. e SEO, N. Changing farm types and irrigation as an adaptation to climate change in Latin American agriculture. World Bank Policy Research Working Paper 4161. Washington, D.C.: World Bank, 2007.

MINISTÉRIO DA INTEGRAÇÃO NACIONAL - MI. A irrigação no Brasil: situação e diretrizes. Brasília: IICA, 2008.

MINISTÉRIO DO MEIO AMBIENTE - MMA. Caderno Setorial de Recursos Hídricos: agropecuária. Brasília: MMA. 2006.

MORTON, J. F. The impact of climate change on smallholder and subsistence agriculture. PNAS, v. 104, n. 50, p. 19697-19704, 2007.

NEGRI, D. H., GOLLEHON, N. R. e AILLERY, M. P. The effects of climatic variability on US irrigation adoption. Climatic Change, v. 69, n. 2-3, p. 299-323, 2005.

NOBRE, C. A., ASSAD, E. D. e OYAMA, M. D. Mudança ambiental no Brasil: o impacto do aquecimento global nos ecossistemas da Amazônia e na agricultura. Scientific American Brasil, v. 80, p. 70-75, 2005.

PINTO, H. S. e ASSAD, E. D. (Eds.). Aquecimento global e cenários futuros da agricultura brasileira. Campinas: Embrapa / Unicamp. 2008.

ROSENBAUM, P. R. e RUBIN D. B. The Central role of the Propensity Score in observational studies for causal effects. Biometrika, v. 70, n. 1, p. 41-55, 1983.

SANGHI, A., ALVES, D., EVERSON, R. e MENDHELSOHN, R. Global warming impacts on Brazilian agriculture: estimates of the Ricardian Model. Economia Aplicada, v. 1, n. 1, p. 7-33, 1997.

SCHLENKER, W., HANEMANN, W. M. e FISHER, A. C. Will U.S. agriculture really benefit from global warming? Accounting for irrigation in the hedonic approach. The American Economic Review, v. 95, n. 1, p. 395-406, 2005.

SEO, N. A microeconometric analysis of adapting portfolios to climate change: adoption of agricultural systems in Latin America. Applied Economic Perspectives and Policy, v. 32, n. 3, p. 489-514, 2010.

SEO, N. An analysis of public adaptation to climate change using agricultural water schemes in South America. Ecological Economics, v. 70, n. 4, p. 825-834, 2011.

SEO, N. e MENDELSOHN, R. A Ricardian analysis of the impact of climate change on South American farms. Chilean Journal Of Agricultural Research, v. 68, n.1, p. 69-79, 2008a.

SEO, N. e MENDELSOHN, R. An analysis of crop choice: adapting to climate change in South American farms. Ecological Economics, v. 67, n. 1, p. 109-116, 2008 b.

SEO, N. e MENDELSOHN, R. Measuring impacts and adaptation to climate change: a structural Ricardian model of African livestock management. Agricultural Economics, v. 38, n. 2, p. 151-165, 2008c.

SIQUEIRA, O. J. F., FARIAS, J. R. B. e SANS, L. M. A. Efeitos potenciais de mudanças climáticas globais na agricultura brasileira e estudos de adaptação para trigo milho e soja. Revista Brasileira de Agrometeorologia, v. 2, n. 1, p. 115-129, 1994. 


\section{Anexos}

Tabela A1. Estimativas das concentrações acumuladas de GEE's no período de 1990 a 2100

\begin{tabular}{cccc}
\hline \multirow{2}{*}{ Cenário } & \multicolumn{3}{c}{ Gases de Efeito Estufa } \\
\cline { 2 - 4 } & Dióxido de Carbono $[\mathrm{CO} 2(\mathrm{Gt})]$ & Metano $\left[\mathrm{CH}_{4}(\mathbf{M t})\right]$ & Óxido Nitroso $\left[\mathbf{N}_{2} \mathbf{O}(\mathbf{M t})\right]$ \\
\hline A1B & $13,5(13,5-17,9)$ & $289(289-640)$ & $7,0(5,8-17,2)$ \\
A2 & $29,1(16,9-34,5)$ & $889(549-1069)$ & $16,5(8,1-19,3)$ \\
\hline
\end{tabular}

Notas: (1) Gt e Mt indicam, respectivamente, gigatoneladas e megatoneladas; (2) Os valores entre parênteses indicam intervalos de variação.

Fonte: IPCC.

Tabela A2. Coeficiente de correlação entre as variáveis utilizadas no estudo

\begin{tabular}{|c|c|c|c|c|c|c|c|c|c|c|c|c|c|c|c|c|c|c|}
\hline Variáveis & 1 & 2 & 3 & 4 & 5 & 6 & 7 & 8 & 9 & 10 & 11 & 12 & 13 & 14 & 15 & 16 & 17 & 18 \\
\hline 1 & 1,00 & & & & & & & & & & & & & & & & & \\
\hline 2 & 0,99 & 1,00 & & & & & & & & & & & & & & & & \\
\hline 3 & $-0,48$ & $-0,49$ & 1,00 & & & & & & & & & & & & & & & \\
\hline 4 & 0,88 & 0,88 & $-0,30$ & 1,00 & & & & & & & & & & & & & & \\
\hline 5 & 0,86 & 0,87 & $-0,30$ & 0,99 & 1,00 & & & & & & & & & & & & & \\
\hline 6 & 0,04 & 0,04 & $-0,35$ & $-0,13$ & $-0,10$ & 1,00 & & & & & & & & & & & & \\
\hline 7 & $-0,53$ & $-0,53$ & 0,12 & $-0,83$ & $-0,81$ & 0,32 & 1,00 & & & & & & & & & & & \\
\hline 8 & 0,11 & 0,12 & 0,52 & 0,38 & 0,40 & $-0,16$ & $-0,43$ & 1,00 & & & & & & & & & & \\
\hline 9 & 0,05 & 0,05 & $-0,11$ & 0,02 & 0,03 & 0,16 & 0,08 & $-0,03$ & 1,00 & & & & & & & & & \\
\hline 10 & 0,14 & 0,14 & $-0,14$ & 0,09 & 0,09 & $-0,03$ & $-0,03$ & $-0,05$ & 0,03 & 1,00 & & & & & & & & \\
\hline 11 & $-0,40$ & $-0,40$ & 0,29 & $-0,35$ & $-0,34$ & 0,00 & 0,20 & $-0,03$ & $-0,01$ & $-0,40$ & 1,00 & & & & & & & \\
\hline 12 & $-0,07$ & $-0,07$ & $-0,05$ & $-0,08$ & $-0,08$ & $-0,01$ & 0,06 & $-0,10$ & $-0,04$ & 0,13 & $-0,21$ & 1,00 & & & & & & \\
\hline 13 & $-0,10$ & $-0,10$ & 0,01 & $-0,18$ & $-0,17$ & 0,10 & 0,23 & $-0,12$ & 0,50 & $-0,03$ & 0,03 & 0,00 & 1,00 & & & & & \\
\hline 14 & $-0,07$ & $-0,07$ & 0,03 & $-0,12$ & $-0,12$ & 0,06 & 0,17 & $-0,08$ & 0,61 & $-0,03$ & 0,04 & $-0,03$ & 0,72 & 1,00 & & & & \\
\hline 15 & 0,58 & 0,60 & $-0,76$ & 0,64 & 0,64 & 0,11 & $-0,55$ & $-0,08$ & 0,08 & 0,09 & $-0,31$ & 0,00 & $-0,13$ & $-0,11$ & 1,00 & & & \\
\hline 16 & $-0,06$ & $-0,06$ & $-0,10$ & $-0,14$ & $-0,13$ & 0,17 & 0,27 & $-0,12$ & 0,72 & 0,01 & $-0,01$ & $-0,01$ & 0,62 & 0,50 & $-0,03$ & 1,00 & & \\
\hline 17 & 0,37 & 0,38 & $-0,14$ & 0,35 & 0,38 & 0,46 & $-0,18$ & 0,33 & 0,11 & $-0,02$ & $-0,09$ & $-0,07$ & 0,00 & 0,00 & 0,22 & $-0,01$ & 1,00 & \\
\hline 18 & $-0,43$ & $-0,45$ & 0,25 & $-0,34$ & $-0,35$ & $-0,24$ & 0,13 & $-0,08$ & $-0,06$ & $-0,02$ & 0,14 & 0,05 & 0,02 & 0,04 & $-0,29$ & 0,01 & $-0,36$ & 1,00 \\
\hline
\end{tabular}

Nota: (1) Temperatura Verão; (2) Temperatura Verão; (3) Precipitação Verão; (4) Temperatura Inverno; (5) Temperatura Inverno ${ }^{2}$; (6) Precipitação Inverno; (7) Variabilidade Temperatura; (8) Variabilidade Precipitação; (9) Recursos Hídricos; (10) Alto Potencial Agrícola; (11) Baixo Potencial Agrícola; (12) Potencial Erosão; (13) Acesso internet; (14) Ensino Superior; (15) Nordeste; (16) Pronaf; (17) Baixa altitude; (18) Alta altitude.

Fonte: Resultados da pesquisa. 
386 - Irrigação como Estratégia de Adaptação de Pequenos Agricultores às Mudanças Climáticas: aspectos econômicos

Tabela A3. Teste de sensibilidade do efeito de tratamento por meio dos limites de Rosenbaum

\begin{tabular}{ccc}
\hline Variável & $\Gamma$ & P-valor crítico \\
\hline & 1,0 & 0,0000 \\
& 1,1 & 0,0000 \\
Valor Terra & 1,2 & 0,0000 \\
& 1,3 & 0,0000 \\
& 1,4 & 0,0000 \\
& 1,5 & 0,0000 \\
\hline
\end{tabular}

Fonte: Resultados da pesquisa.

Tabela A4. Desvios padrão entre os valores da terra simulados para irrigantes e produtores de sequeiro em diferentes períodos de tempo e cenários climáticos

\begin{tabular}{cccc}
\hline \multirow{2}{*}{ Período } & Sistema produtivo & \multicolumn{2}{c}{ Cenários } \\
\cline { 3 - 4 } & & $A 1 B$ & $A 2$ \\
\hline \multirow{2}{*}{$2020-2080$} & Irrigante & 5,80 & 2,69 \\
& Sequeiro & 57,83 & 71,86 \\
\multirow{2}{*}{$2020-2050$} & Irrigante & 2,29 & 0,50 \\
& Sequeiro & 43,83 & 11,13 \\
& Irrigante & 5,67 & 3,02 \\
$2050-2080$ & Sequeiro & 37,87 & 93,06 \\
\hline
\end{tabular}

Fonte: Resultados da pesquisa. 